\title{
Combining monojet, supersymmetry, and dark matter searches
}

\author{
Alexandre Arbey ${ }^{\dagger}$ \\ Centre de Recherche Astrophysique de Lyon, Observatoire de Lyon, Saint-Genis Laval Cedex, F-69561, \\ France; CNRS, UMR 5574; Ecole Normale Supérieure de Lyon, France; Université de Lyon, \\ Université Lyon 1, F-69622 Villeurbanne Cedex, France; and CERN, CH-1211 Geneva, Switzerland \\ Marco Battaglia* \\ University of California at Santa Cruz, Santa Cruz Institute of Particle Physics, California 95064, \\ USA and CERN, CH-1211 Geneva, Switzerland \\ Farvah Mahmoudi ${ }^{*}$ \\ Clermont Université, Université Blaise Pascal, CNRS/IN2P3, LPC, BP 10448, \\ F-63000 Clermont-Ferrand, France and CERN, CH-1211 Geneva, Switzerland
}

(Received 18 December 2013; published 7 April 2014)

\begin{abstract}
This paper presents new results on the combined sensitivity of the LHC and underground dark matter search experiments to the lightest neutralino as a weakly interacting massive particle candidate in the minimal supersymmetric extension of the standard model. We show that monojet searches significantly extend the sensitivity to the neutralino mass in scenarios where scalar quarks are nearly degenerate in mass with it. The inclusion of the latest bound by the LUX experiment on the neutralino-nucleon spinindependent scattering cross section expands this sensitivity further, highlighting the remarkable complementarity of jets $/ \ell \mathrm{s}+$ missing transverse energy and monojets at the LHC, and dark matter searches in probing models of new physics with a dark matter candidate. The qualitative results of our study remain valid after accounting for theoretical uncertainties.
\end{abstract}

DOI: 10.1103/PhysRevD.89.077701

Astrophysical data have convincingly established the existence of nonbaryonic dark matter (DM), most likely due to a new kind of neutral, stable, weakly interacting massive particle (WIMP) [1]. One of the most appealing features of supersymmetry (SUSY) with conserved $R$ parity is that it provides us with a natural candidate for WIMP dark matter [2-6]. If the lightest supersymmetric particle (LSP) in the theory is the lightest neutralino $\tilde{\chi}_{1}^{0}$, it represents a WIMP with the appropriate properties to account for the observed cosmic dark matter. A $\tilde{\chi}_{1}^{0}$ with mass of $\mathcal{O}(100 \mathrm{GeV})$ and typical weak-interaction couplings develops a relic density in the Universe of the same order as the value now precisely measured by satellite experiments from the analysis of the cosmic microwave background (CMB) spectrum $[7,8]$. The search for WIMPs has become one of the most compelling research areas at the intersection between collider physics, nonaccelerator particle physics and cosmology [9]. In fact, a WIMP neutralino can be searched for at the LHC, first in direct SUSY searches, from the decay chains of heavier SUSY particles through topologies with the production of Standard Model (SM) particles and significant missing transverse energy (MET), then in direct $\tilde{\chi} \tilde{\chi}$ pair production through "monojet" events. Direct DM searches can reveal signals of cosmic WIMP

\footnotetext{
*Corresponding author.

MBattaglia@lbl.gov

†alexandre.arbey@ens-lyon.fr

*mahmoudi@in2p3.fr
}

PACS numbers: 12.60.Jv, 14.80.Ly, 14.80.Nb, 95.35.+d

neutralinos through the small energy released in their scattering on the nucleons of the sensitive detector volume in underground experiments. All these searches are characterized by different sensitivities, depending on the SUSY parameters. Within a well-defined model, these sensitivities can be compared and their complementarity and redundancy studied. In the study of WIMP neutralinos, the phenomenological minimal supersymmetric extension of the SM (pMSSM), a minimal, $R$ parity conserving SUSY model with 19 free parameters [10], represents a very practical model providing a broad and unbiased perspective on the generic MSSM phenomenology. Its parametrization ensures that the SUSY particle masses are independent, while keeping a small enough number of parameters limited to allow full scans of the model phase space to be performed. A comparison of the jets + MET and monojet searches at the LHC was already presented in [11], where the $7 \mathrm{TeV}$ data and specific masses were considered. An earlier general study of neutralinos as DM candidates in the pMSSM was discussed in [12]. We have presented results for specific pMSSM scenarios with a light WIMP neutralino in $[13,14]$, and we shall not discuss them here. In this paper, we report the results of the first study of WIMP neutralino sensitivity in the framework of the pMSSM, which includes the LHC jets/leptons + MET and the important monojet analyses on full $8 \mathrm{TeV}$ data, the PLANCK CMB constraints and the new results on direct DM searches from the LUX experiment. We show how the inclusion of the LHC monojet analyses and the 
DM direct detection bounds significantly expands the region of MSSM parameters probed by the data. These searches can test regions of the parameter space not accessible to the LHC-direct SUSY searches, characterized by specific kinematics of signal events, in particular, with SUSY particles having masses almost degenerate with the LSP $\tilde{\chi}$. We develop a new methodology to interpret the monojet search results in SUSY in a largely modelindependent way. The complementarity with jet + MET direct SUSY searches at the LHC and direct dark matter experiments proves to be remarkable. These conclusions hold also when we account for QCD uncertainties in the monojet event cross section and astrophysical uncertainties in the derivation of the $\tilde{\chi}-p$ scattering cross section, $\sigma_{\tilde{\chi} p}^{\mathrm{SI}}$.

This study is based on a large statistics scan of the pMSSM parameters, constraints from lower-energy data, and the determination of the sensitivity of various search analyses to the spectrum and decay pattern of each accepted pMSSM point. The tools used to perform the scans and the analysis have been presented in $[15,16]$. SUSY particle spectra are calculated using SOFTSUSY 3.2.3 [17], the decay branching fractions for the Higgs and SUSY particles with HDECAY 5.10 [18] and SDECAY [19], respectively. Particularly relevant to this study are the calculations of the neutralino scattering cross sections and relic density, performed with micrOMEGAs [20] and SuperIso Relic v3.2 [21,22], respectively. A total of $\sim 10^{7}$ valid pMSSM points have been simulated. Of these, $1.15 \mathrm{M}$ are accepted after imposing the constraints from electroweak, flavor, DM relic density and lower-energy data discussed in [23]. The relic DM density constraint is applied in the loose form by taking the upper limit from the PLANCK data [8], but allowing other particles to contribute to the observed cosmic DM and/or modifications to the early universe properties, corresponding to $10^{-4}<\Omega_{\chi} h^{2}<0.163$. In order to test the compatibility of the accepted pMSSM points with the LHC searches, we simulate event samples and perform a parametric simulation for the event reconstruction. Events are generated with MadGraph 5 [24] and Pythia 8.150 [25] with the CTEQ6L1 parton distribution functions (PDFs) [26], and the physics observables are obtained from fast simulation performed using Delphes 3.0 [27]. Signal selection cuts are applied to simulated signal events, while the number of SM background events in the signal regions are from the experiment estimates. The 95\% confidence level (C.L.) exclusion of each SUSY point in the presence of background only is determined using the C.L. method [28].

The monojet searches are based on the process $p p \rightarrow \tilde{\chi} \tilde{\chi}+j$, which can be seen as a complementary process to the $\tilde{\chi}+p \rightarrow \tilde{\chi}+p$ scattering process of DM direct-detection experiments, since they both probe the WIMP coupling with standard matter. Monojets are a distinctive topology of events with a single, high $p_{t}$ hadronic jet, limited additional hadronic activity, and large MET. Their relevance to the search of pair production of weakly interacting, or noninteracting, particles was first exploited at the Tevatron [29], and they are now actively searched for at the LHC by ATLAS [30] and CMS [31], with the analysis of $\sim 20 \mathrm{fb}^{-1}$ of data at $8 \mathrm{TeV}$. The bounds from monojet searches can be interpreted as exclusion contours of the spin-independent WIMP scattering cross section $\sigma_{\tilde{\chi}}^{\mathrm{SI}}$, as a function of the WIMP mass to compare with DM direct searches, relying on an effective approach with contact operators [32,33]. However, its validity is limited to the cases where a single heavy particle mediates the WIMP scattering with the nucleons. While this condition applies to simple DM models involving just one heavy mediator and a single new particle in the final states, it is generally not applicable to more complex models with several particles in the interaction, such as SUSY. In the specific case of the MSSM with a neutralino LSP studied here, monojet events originate from the channel $p p \rightarrow \tilde{\chi}_{1}^{0} \tilde{\chi}_{1}^{0}+j$, mediated at tree level by $Z$ and Higgs bosons in $s$ channel and SUSY particles in $t$ channel, but also from processes such as $p p \rightarrow \tilde{q} \overline{\tilde{q}}+j$ or $p p \rightarrow \tilde{g} \tilde{g}+j$, in particular, if SUSY particles in the final state decay to a $\tilde{\chi}$ and a soft quark or gluon. Such events are of importance when the splitting between the mass of the SUSY strongly interacting particles with the LSP is small and these events make monojet searches especially valuable and complementary to the jets + MET channels in these scenarios. The impact of the monojet searches at Tevatron and the LHC in the MSSM has already been studied in simple, or simplified, models where only one scalar quark or gluino is close in mass to the lightest neutralino [11,34-47]. In order to study the monojet searches in the more general cases offered by the pMSSM, where many SUSY strongly interacting particles can have similar mass values, we simulate monojet events with MadGraph 5, generating the full $2 \rightarrow 3$ matrix elements for all the possible processes $p p \rightarrow \tilde{g} / \tilde{q} / \tilde{\chi}_{1}^{0}+\tilde{g} / \tilde{q} / \tilde{\chi}_{1}^{0}+j$, interfaced with the Pythia 6 parton shower [48]. This method, specifically developed for our study, appears appropriate for the interpretation of monojet results in the context of general SUSY models. Because of computing time limitations, we generate the processes involving the LSP neutralino, the lightest of the SUSY strongly interacting particles (LSSP) and additional channels involving the other SUSY particles close in mass to the LSP or the LSSP. We verify that the inclusion of the heavier SUSY states does not significantly modify our results, on a selected set of pMSSM points, where all SUSY processes are generated.

MET searches at the LHC have considered a wide variety of topologies and final states. Here, we consider the preliminary results by the ATLAS Collaboration for the search of scalar quarks of the first two generations and gluinos in the jets + MET channel [49], of scalar top and bottom quarks with $b$-tagged jets, and MET [50] and chargino/neutralino associate production in the two-[51] and three - leptons + MET channels [52].

Searches for WIMP neutralinos scattering in direct DM detection experiments probe the same physical vertex as some of the LHC searches. This makes the comparison of 
the reach and implications of these searches in the pMSSM particularly interesting. Several experiments have recently reported results corresponding to sensitivities relevant to the study of MSSM scenarios. The most recent result, from LUX, a dual-phase Xenon time projection chamber underground experiment [53], reported a minimum value of the $95 \%$ C.L. upper limit of $\sigma_{\tilde{\chi} p}^{\mathrm{SI}}=10^{-9} \mathrm{pb}$ for a WIMP mass of $\sim 40 \mathrm{GeV}$. The typical range for MSSM points is $10^{-14}<$ $\sigma_{\tilde{\chi} p}^{\mathrm{SI}}<10^{-6} \mathrm{pb}$ for $100<M_{\tilde{\chi}}<1000 \mathrm{GeV}$ [23]. The Xenon-100 [54] and LUX results challenge the excesses of events reported by several other experiments [55-58], which may be interpreted as due to a low-mass WIMP with large scattering cross section. We consider the effect of these bounds on the pMSSM parameters by imposing the 95\% C.L. upper limit on $\sigma_{\tilde{\chi} p}^{\text {SI }}$ obtained by LUX on our scan points. Similar results are obtained using the Xenon-100 limit.

The sensitivity of the searches discussed above is quantified first by studying the fraction of accepted pMSSM points incompatible with a given channel or combination of channels at 95\% C.L. $(F)$. Results are summarized in Table I. About two-thirds of the accepted pMSSM points, compatible with the loose $\Omega h^{2}$ relic density constraint, are rejected by the combination of the LHC and LUX bounds considered here. As anticipated, the inclusion of the monojet and DM direct search ensures the exclusion of a significant fraction of points, not excluded by the jets/leptons + MET LHC searches, corresponding to 17\% of our accepted pMSSM points. Contrary to the effective operator approach that would predict only of order $2 \times 10^{-4}$ of the points to be excluded by the monojet bounds, the analysis of the $2 \rightarrow 3$ SUSY processes for our pMSSM points gives $26 \%$ of these rejected by the LHC monojet analyses. Then, we study our results in terms of the fraction of accepted pMSSM points in our scans, excluded by the jets/leptons/monojet + MET LHC and the direct DM searches discussed above, as a function of a single (or a pair of) pMSSM parameter(s) to study the parameter regions which benefit from the inclusion of the monojet and direct DM searches. In the context of this study, the parameters of interest are the mass of the WIMP candidate,

TABLE I. Fraction $F$ of pMSSM points rejected by the various LHC channels and the direct DM searches. The effect of systematic uncertainties is also given.

\begin{tabular}{lcc}
\hline \hline Search & \multicolumn{2}{c}{ Fraction of pMSSM points } \\
Excluded & Excluded uniquely \\
\hline Jets + MET & 0.467 & \\
$b-$ jets + MET & 0.178 & \\
$\ell$ s + MET & 0.019 & \\
All jets $/ \ell$ s + MET & $0.483 \pm 0.016$ & \\
monojet + MET & $0.262 \pm 0.020$ & 0.045 \\
All LHC MET Searches & $0.541 \pm 0.018$ & 0.300 \\
DM searches & $0.335_{-0.120}^{+0.090}$ & 0.120 \\
All searches & $0.673_{-0.047}^{+0.032}$ & \\
\hline \hline
\end{tabular}

the neutralino LSP $\left(M_{\tilde{\chi}^{0}}\right)$, the LSSP mass $\left(M_{\tilde{q}, \tilde{g}}\right)$ and the mass splitting $\left(\Delta M=M_{\tilde{q}, \tilde{g}}-M_{\tilde{\chi}}\right)$. In general, the masses determine the production cross section at the LHC, while $\Delta M$ controls the signal event kinematics and thus the selection efficiency. In particular, we are interested in assessing the exclusion reach of the LHC and direct DM searches in the $\sigma_{\tilde{\chi} p}^{\mathrm{SI}}-M_{\tilde{\chi}}$ and $M_{\tilde{q}, \tilde{g}}-M_{\tilde{\chi}}$ planes and contrast the sensitivity of the various channels, identifying specific regions of the parameter space which are unique to the monojet analyses and the direct DM searches. First we consider the parameter plane $\sigma_{\tilde{\chi} p}^{\mathrm{SI}}-M_{\tilde{\chi}}$ where the comparison to the bounds from direct detection experiments is straightforward (see Fig. 1). The sensitivity of the LHC monojet searches provides a bound which extends to larger WIMP- $\tilde{\chi}$ masses along a line of almost constant $\sigma_{\tilde{\chi} p}^{\text {SI }}$ compared to the jets + MET analyses. The analysis of the $M_{\tilde{q}, \tilde{g}}-M_{\tilde{\chi}}$ plane provides us with a good illustration of the jets/lepton + MET, monojet and direct DM search complementarity. Figures 2 and 3 shows the fraction of points excluded at $95 \%$ C.L. by the combination of all these searches and the contours enclosing the region where $68 \%$ of the pMSSM points are rejected by jets/lepton+MET channels and their combination with monojets. The latter channel, with its specific sensitivity to low-mass SUSY particles, in particular, in degenerate scenarios due to the buildup of the monojet cross section, significantly improves the rejection of points along the $M_{\tilde{q}, \tilde{g}} \simeq M_{\tilde{\chi}}$ line, where the jets + MET searches are weaker due to the reduced transverse momentum of the hadronic jets. This effect shows a gain of $\sim 80 \mathrm{GeV}$ in sensitivity to the neutralino LSP mass close to the limit $M_{\tilde{q}, \tilde{g}} \simeq M_{\tilde{\chi}}$ and a $15 \%$ wider surface covered by the contour. The gain is further enhanced with the inclusion of the LUX bound by $\sim 120 \mathrm{GeV}$ in sensitivity to the WIMP $\tilde{\chi}$ mass and a $62 \%$ wider surface. The extent of these gains is reduced when requiring larger fractions of pMSSM points to be rejected. The surface covered by the contour enclosing the region with $90 \%$ of the pMSSM points rejected increases by only $8 \%$ with the addition of the monojets and by $30 \%$ with that
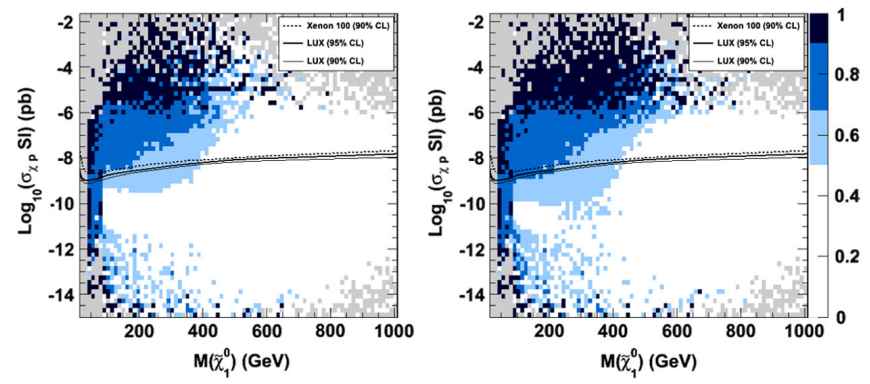

FIG. 1 (color online). Fraction of pMSSM points excluded by jets/leptons + MET (left panel) and their combination with monojet searches (right panel) in the $\sigma_{\tilde{\chi} p}^{\mathrm{SI}}-M_{\tilde{\chi}}$ parameter plane. The upper limits from direct detection experiments are also indicated. 
BRIEF REPORTS

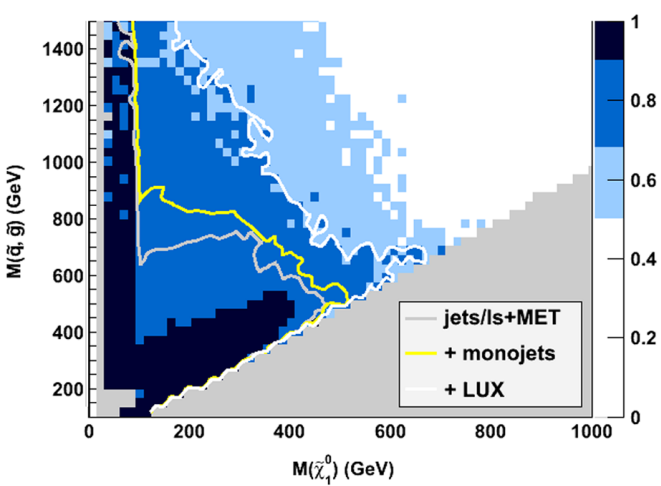

FIG. 2 (color online). Fraction of pMSSM points excluded by the combination of the LHC jets/leptons+MET, monojet analyses and direct DM searches in the $M_{\tilde{q}, \tilde{g}}-M_{\tilde{\chi}}$ parameter plane. The lines give the parameter region where $68 \%$ of the pMSSM points are excluded by the jets/leptons + MET searches alone (grey line), the combination with monojet searches (yellow line) and also with the LUX experiment (white line).

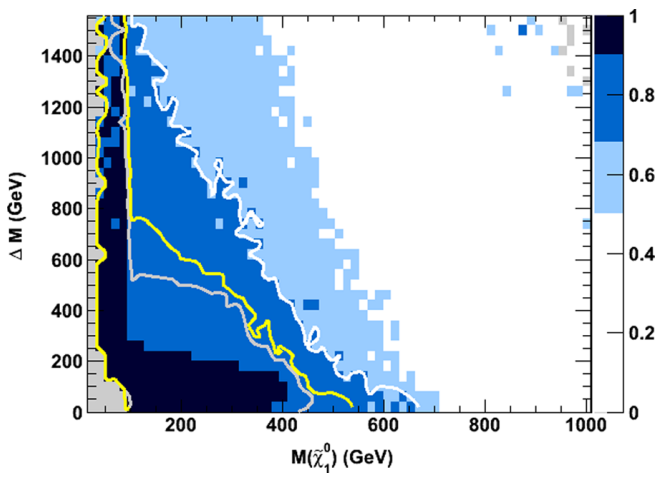

FIG. 3 (color online). Fraction of pMSSM points excluded by the combination of the LHC jets/leptons + MET, monojet analyses and direct DM searches in the $\Delta M-M_{\tilde{\chi}}$ parameter plane. The conventions for the lines are as in Fig. 2.

of the LUX bounds. The fraction of pMSSM points excluded at $95 \%$ C.L. by the LHC and DM searches is shown as a function of the $\tilde{\chi}$ mass and $\Delta M$, when integrating over all other pMSSM parameters in Fig. 4. The distribution is rather smooth in the $\tilde{\chi}$ mass value, but the increase in sensitivity at very small and intermediate-tolarge $\Delta M$ values afforded by the inclusion of the monojet and DM search limits is evident. The fraction of excluded points depends on the range of parameters used in the scans. We estimate this effect by expanding the range of the $\tilde{g}$ mass from 2.5 to $3.5 \mathrm{TeV}$. This decreases correspondingly the fraction of points excluded by the combination of the LHC searches by $29 \%$ and that excluded by the LUX result by $20 \%$. Finally, we estimate the effect of systematic uncertainties by varying the production cross sections of $p p \rightarrow \tilde{q} \tilde{q}, \tilde{g} \tilde{g}$ by $\pm 20 \%$ to account for PDF uncertainties [59], and those of $p p \rightarrow \tilde{\chi}_{1}^{0} \tilde{\chi}_{1}^{0}+j, \tilde{q} \tilde{q}+j$ by $\pm 30 \%$ to include uncertainties from the generation [11]. However,
PHYSICAL REVIEW D 89, 077701 (2014)
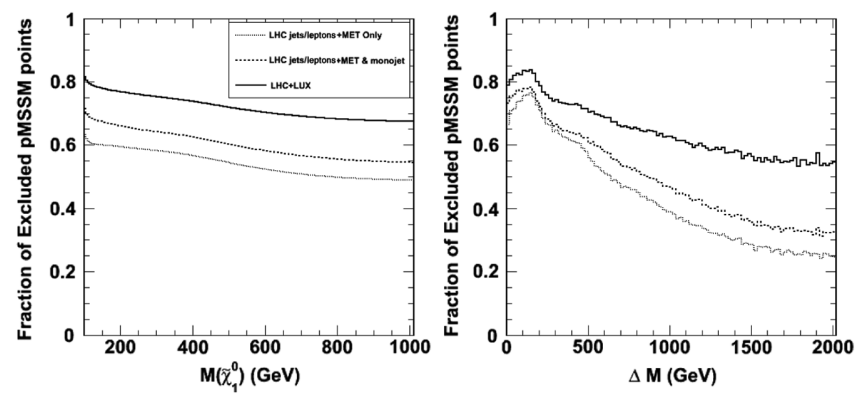

FIG. 4. Fraction of pMSSM points excluded by the jets/leptons + MET (dotted line), their combination with the monojet analyses (dashed line), and that with the LUX direct DM search (continuous line) as a function of $M_{\tilde{\chi}}$ (left panel) and $\Delta M$ (right panel).

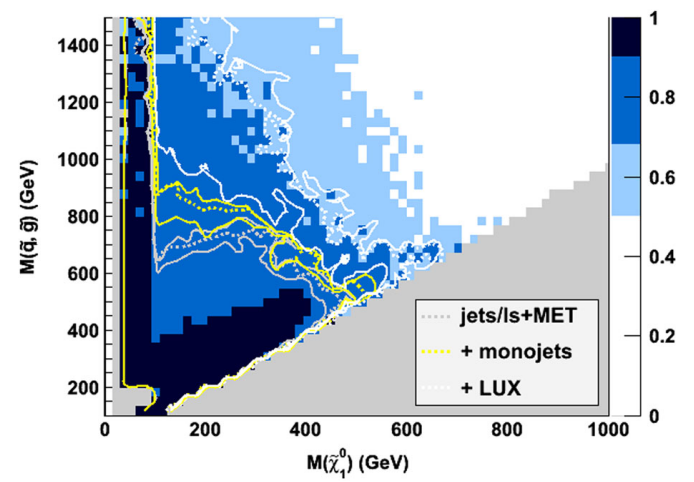

FIG. 5 (color online). Ranges of the contours as given in Fig. 2, obtained by modifying the production and the scattering cross sections to account for systematic uncertainties, as discussed in the text. The contours corresponding to the values used for Fig. 2 are given by the dotted lines.

their effects are rather small (see Table I). The $\sigma_{\tilde{\chi} p}^{\text {SI bounds }}$ from direct-detection DM experiments depend on assumptions on the local DM density in the Galaxy. We propagate the uncertainty on the local DM density $\rho=(0.3 \pm$ $0.1) \mathrm{GeV} / \mathrm{cm}^{3}$ [60] to the $\sigma_{\tilde{\chi} p}^{\mathrm{SI}} 95 \%$ C.L. bound. Their effect in the $M_{\tilde{q}, \tilde{g}}-M_{\tilde{\chi}}$ parameter plane are summarized in Fig. 5, which shows that the qualitative results of our study remain valid after accounting for these theoretical uncertainties.

The authors are grateful to several colleagues for discussions on the topics presented in this paper. In particular, R. Gaitskell and J. Chapman provided the LUX results in numerical form, B. Fuks advised us on the monojet cross section calculations, and C. Wagner engaged in useful discussion. We also acknowledge the LPCC and the CERN PH-TH unit for computing support. The work of A. A. was supported by the Fédération de Recherche A.-M. Ampère de Lyon; that of F. M. was supported, in part, by the National Science Foundation under Grant No. PHYS1066293, and through the hospitality of the Aspen Center for Physics. 
[1] G. Bertone, D. Hooper, and J. Silk, Phys. Rep. 405, 279 (2005).

[2] H. Pagels and J. R. Primack, Phys. Rev. Lett. 48, 223 (1982).

[3] S. Weinberg, Phys. Rev. Lett. 48, 1303 (1982).

[4] S. Weinberg, Phys. Rev. Lett. 50, 387 (1983).

[5] J. Ellis, J. S. Hagelin, D. V. Nanopoulos, K. Olive, and M. Srednicki, Nucl. Phys. B238, 453 (1984).

[6] G. Jungman, M. Kamionkowski, and K. Griest, Phys. Rep. 267, 195 (1996).

[7] G. Hinshaw et al. (WMAP Collaboration), Astrophys. J. Suppl. Ser. 208, 19 (2013).

[8] P. A. R. Ade et al. (PLANCK Collaboration), arXiv: 1303.5076.

[9] E. A. Baltz, M. Battaglia, M. E. Peskin, and T. Wizansky, Phys. Rev. D 74, 103521 (2006).

[10] A. Djouadi et al. (MSSM Working Group Collaboration), arXiv:hep-ph/9901246.

[11] H. Dreiner, M. Krämer, and J. Tattersall, Phys. Rev. D 87, 035006 (2013).

[12] M. Cahill-Rowley et al., arXiv:1305.6921.

[13] A. Arbey, M. Battaglia, and F. Mahmoudi, Eur. Phys. J. C 72, 2169 (2012).

[14] A. Arbey, M. Battaglia, and F. Mahmoudi, Phys. Rev. D 88, 095001 (2013).

[15] A. Arbey, M. Battaglia, and F. Mahmoudi, Eur. Phys. J. C 72, 1847 (2012).

[16] A. Arbey, M. Battaglia, and F. Mahmoudi, Eur. Phys. J. C 72, 1906 (2012).

[17] B. C. Allanach, Comput. Phys. Commun. 143, 305 (2002).

[18] A. Djouadi, J. Kalinowski, and M. Spira, Comput. Phys. Commun. 108, 56 (1998).

[19] M. Muhlleitner, A. Djouadi, and Y. Mambrini, Comput. Phys. Commun. 168, 46 (2005).

[20] G. Belanger, F. Boudjema, A. Pukhov, and A. Semenov, Comput. Phys. Commun. 180, 747 (2009).

[21] F. Mahmoudi, Comput. Phys. Commun. 178, 745 (2008); 1801579 (2009).

[22] A. Arbey and F. Mahmoudi, Comput. Phys. Commun. 181, 1277 (2010).

[23] A. Arbey, M. Battaglia, A. Djouadi, and F. Mahmoudi, Phys. Lett. B 720, 153 (2013).

[24] J. Alwall, M. Herquet, F. Maltoni, O. Mattelaer, and T. Stelzer, J. High Energy Phys. 06 (2011) 128.

[25] T. Sjöstrand, S. Mrenna, and P. Skands, J. High Energy Phys. 05 (2006) 026; Comput. Phys. Commun. 178, 852 (2008).

[26] J. Pumplin, D. R. Stump, J. Huston, H.-L. Lai, P. Nadolsky, and W.-K. Tung, J. High Energy Phys. 07 (2002) 012.

[27] S. Ovyn, X. Rouby, and V. Lemaitre, arXiv:0903.2225; J. Favereau, C. Delaere, P. Demin, A. Giammanco, V. Lemaître, A. Mertens, and M. Selvaggi, J. High Energy Phys. 02 (2014) 057.

[28] A. L. Read, J. Phys. G 28, 2693 (2002).

[29] S. Chatrchyan et al. (CMS Collaboration), J. High Energy Phys. 09 (2012) 094.
[30] ATLAS Collaboration, Note ATLAS-CONF-2013-068.

[31] CMS Collaboration, Note CMS PAS EXO-12-048.

[32] J. Goodman, M. Ibe, A. Rajaraman, W. Shepherd, T. M. P. Tait, and H.-B. Yu, Phys. Lett. B 695, 185 (2011).

[33] J. Goodman, M. Ibe, A. Rajaraman, W. Shepherd, T. M. P. Tait, and H.-B. Yu, Phys. Rev. D 82, 116010 (2010).

[34] J. F. Gunion and S. Mrenna, Phys. Rev. D 62, 015002 (2000).

[35] J. Alwall, M.-P. Le, M. Lisanti, and J. G. Wacker, Phys. Lett. B 666, 34 (2008).

[36] M. Carena, A. Freitas, and C. E. M. Wagner, J. High Energy Phys. 10 (2008) 109.

[37] J. Alwall, M.-P. Le, M. Lisanti, and J. G. Wacker, Phys. Rev. D 79, 015005 (2009).

[38] E. Izaguirre, M. Manhart, and J. G. Wacker, J. High Energy Phys. 12 (2010) 030.

[39] T. J. LeCompte and S. P. Martin, Phys. Rev. D 84, 015004 (2011).

[40] M. A. Ajaib, T. Li, and Q. Shafi, Phys. Rev. D 85, 055021 (2012).

[41] T. J. LeCompte and S. P. Martin, Phys. Rev. D 85, 035023 (2012).

[42] B. He, T. Li, and Q. Shafi, J. High Energy Phys. 05 (2012) 148.

[43] M. Drees, M. Hanussek, and J. S. Kim, Phys. Rev. D 86, 035024 (2012).

[44] H. K. Dreiner, M. Kramer, and J. Tattersall, Europhys. Lett. 99, 61001 (2012).

[45] B. Bhattacherjee and K. Ghosh, arXiv:1207.6289.

[46] Z.-H. Yu, X.-J. Bi, Q.-S. Yan, and P.-F. Yin, Phys. Rev. D 87, 055007 (2013).

[47] C. Han et al., arXiv:1310.4274.

[48] T. Sjostrand, S. Mrenna, and P. Z. Skands, J. High Energy Phys. 05 (2006) 026.

[49] ATLAS Collaboration, Note ATLAS-CONF-2013-047.

[50] ATLAS Collaboration, Note ATLAS-CONF-2013-053.

[51] ATLAS Collaboration, Note ATLAS-CONF-2013-049.

[52] ATLAS Collaboration, Note ATLAS-CONF-2013-035.

[53] D. S. Akerib et al. (LUX Collaboration), arXiv:1310.8214.

[54] E. Aprile et al. (XENON100 Collaboration), Phys. Rev. Lett. 109, 181301 (2012).

[55] R. Bernabei et al. (DAMA/LIBRA Collaborations), Eur. Phys. J. C 67, 39 (2010).

[56] C. E. Aalseth et al. Phys. Rev. Lett. 107, 141301 (2011).

[57] G. Angloher et al., Eur. Phys. J. C 72, 1971 (2012).

[58] R. Agnese et al. (CDMS Collaboration), Phys. Rev. Lett. 111, 251301 (2013).

[59] P. Nadolsky, H.-L. Lai, Q.-H. Cao, J. Huston, J. Pumplin, D. Stump, W.-K. Tung, and C.-P. Yuan, Phys. Rev. D 78, 013004 (2008).

[60] J. Bovy and S. Tremaine, Astrophys. J. 756, 89 (2012). 